\title{
Erratum
}

\section{Erratum: Trinh et al., "Cellular and Network Mechanisms May Generate Sparse Coding of Sequential Object Encounters in Hippocampal-Like Circuits"}

In the article "Cellular and Network Mechanisms May Generate Sparse Coding of Sequential Object Encounters in Hippocampal-Like Circuits," by Anh-Tuan Trinh, Stephen E. Clarke, Erik Harvey-Girard, and Leonard Maler, which published online on July 19, 2019, the legend for Extended Data Figure 8-1 was duplicated from the legend for Extended Data Figure 5-1 because of a production error. The legend should instead read:

Current-evoked spiking decreases the AHP amplitude of DL neuron. $\boldsymbol{A}$, Example trace of a DL neuron's response to a +60 pA current step injection lasting $500 \mathrm{~ms}$. Consecutive spiking causes the AHP amplitude to decrease when compared with the first AHP as emphasized by the arrow. A black dashed line is placed to coincide with the minimum of the first spike's AHP. We hypothesize that the decrease in AHP amplitude is because of a reduction in $\mathrm{Ca}^{2+}$ influx (i.e., $\mathrm{Ca}^{2+}$-dependent $\mathrm{Ca}^{2+}$ channel inactivation) and subsequent reduction in SK channel opening. $\boldsymbol{B}$, The decrease in AHP amplitude between the second and first spikes is plotted as a function of the time interval between the first two spikes similar to Figure $8 B$. Each black square represents a spike pair taken from a trace that did not contain a burst (total of 160 nonburst spike pairs), while each gray triangle represents a spike pair taken from a trace that contained a burst at the beginning of the trace (total of 117 burst spike pairs). The majority of the AHPs are reduced throughout the $300 \mathrm{~ms}$ test period without any evident recovery trend.

Additionally, Itskov et al. (2008) should not have appeared in the reference list; all reference citations should instead be to Itskov et al. (2011). The online version has been corrected. 